\title{
CORRECTION
}

View Article Online

View Journal I View Issue

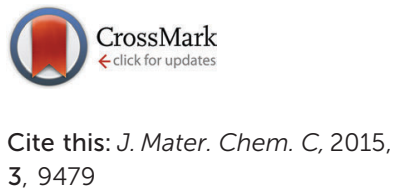

3, 9479

DOI: $10.1039 /$ c5tc90156a

www.rsc.org/MaterialsC

\section{Correction: Ternary donor-acceptor phosphine oxide hosts with peculiar high energy gap for efficient blue electroluminescence}

\author{
Mingzhi Sun, ${ }^{a}$ Liping Zhu, ${ }^{b}$ Wenjing Kan, ${ }^{a}$ Ying Wei, ${ }^{a c}$ Dongge Ma, ${ }^{\star b}$ Xuefeng Fan, ${ }^{b}$ \\ Wei Huang*C and Hui Xu*ac
}

Correction for 'Ternary donor-acceptor phosphine oxide hosts with peculiar high energy gap for efficient blue electroluminescence' by Mingzhi Sun et al., J. Mater. Chem. C, 2015, DOI: 10.1039/c5tc02029h.

An incorrect CCDC reference number appears in the footnote on the first page. The correct CCDC reference number for the single crystal structure of PCImbPO is 1418343. The correct CIF file was uploaded to replace the incorrect one as part of the electronic supplementary information for this article on 21st August 2015.

The Royal Society of Chemistry apologises for these errors and any consequent inconvenience to authors and readers.

\footnotetext{
${ }^{a}$ Key Laboratory of Functional Inorganic Material Chemistry, Ministry of Education, School of Chemistry and Materials, Heilongjiang University, 74 Xuefu Road, Harbin 150080, P. R. China. E-mail: hxu@hlju.edu.cn, iamhxu@njtech.edu.cn

${ }^{b}$ State Key Laboratory of Polymer Physics and Chemistry, Changchun Institute of Applied Chemistry, Chinese Academy of Sciences, 5625 People Street, Changchun 130022, P. R. China. E-mail:mdg1014@ciac.jl.cn

${ }^{c}$ Institute of Advanced Materials, Nanjing Tech University (Nanjing Tech), 30 South Puzhu Road, Nanjing 211800, P. R. China. E-mail: wei-huang@njtech.edu.cn
} 